ÉGYPTE

monde arabe

\section{Égypte/Monde arabe}

$10 \mid 1992$

Partage de l'eau dans le monde arabe

\title{
La sécurité hydrique dans les pays arabes
}

\section{Kamel Kheir et Nizar Akar}

Traducteur : Frédérique Gourdan

\section{(2) OpenEdition \\ Journals}

Édition électronique

URL : https://journals.openedition.org/ema/1412

DOI : 10.4000/ema.1412

ISSN : 2090-7273

Éditeur

CEDEJ - Centre d'études et de documentation économiques juridiques et sociales

Édition imprimée

Date de publication : 30 juin 1992

Pagination : 63-77

ISSN : 1110-5097

\section{Référence électronique}

Kamel Kheir et Nizar Akar, «La sécurité hydrique dans les pays arabes », Égypte/Monde arabe [En ligne], 10 | 1992, mis en ligne le 08 juillet 2008, consulté le 07 juillet 2022. URL : http://

journals.openedition.org/ema/1412; DOI : https://doi.org/10.4000/ema.1412

Ce document a été généré automatiquement le 7 juillet 2022.

Tous droits réservés 


\title{
La sécurité hydrique dans les pays arabes
}

\author{
Kamel Kheir et Nizar Akar
}

Traduction : Frédérique Gourdan

\section{NOTE DE L'ÉDITEUR}

Article paru en arabe dans la revue Chu'ûn 'arabiyya, décembre 1990.

1 S'approvisionner en eau, élément fondamental de la perpétuation de l'espèce humaine et de son développement, a constitué une des principales préoccupations de l'homme tout au long de l'histoire, comme en témoigne la présence de vestiges de regroupements humains en bordure des fleuves et à proximité des sources d'eau douce : la survie et le développement des sociétés étaient liés pour une large part à la capacité et à la permanence de ces sources. Avec la croissance, démographique et l'augmentation de la consommation hydrique dans tous les domaines de la vie, le problème se complique aujourd'hui, à des degrés divers d'une région à l'autre.

2 La question de la sécurité hydrique des pays arabes a pris une importance toute particulière dans la chaîne des priorités; elle est désormais traitée avec le plus grand sérieux pour les raisons qui vont suivre :

- Les pays arabes sont majoritairement situés dans des régions à climat sec et désertique, caractérisé par une faible pluviosité et des températures élevées tout au long de l'année ; le taux d'évaporation peut atteindre, comme dans les Emirats Arabes Unis ${ }^{1}$, 95\%. (...)

- La démographie galopante qui affecte la plupart des pays arabes rend la question encore plus complexe.

- L'augmentation du niveau de vie, principalement dans les pays pétroliers, entraîne une augmentation inconsidérée de la consommation en eau, sans aucun rapport avec les potentialités hydriques de ces pays qui en utilisent des quantités considérables pour leurs puits de pétrole. Ainsi par exemple, l'Arabie Saoudite a utilisé à cet effet, pour la seule année 1981, environ 400 millions de $\mathrm{m}^{32}$. 
- Les plans de développement prévus dans la majorité des pays arabes pour garantir la sécurité alimentaire exigent le développement de l'agriculture et par conséquent l'extension des terres agricoles, donc un accroissement de la demande en eau.

Tableau 1 : Population des pays arabes et consommation domestique de l'eau

Tábleau 1: Population des pays arabes et consommation domestique de feau pour les années 85 et sulv. et prévisions pour les années 2000 et 2015

\begin{tabular}{|c|c|c|c|c|c|c|c|}
\hline & $\begin{array}{l}\text { Nb dhabitants } \\
\text { (en millions) } \\
\text { par année du } \\
\text { recensement }\end{array}$ & $\begin{array}{l}\text { Consom. } \\
\text { domest. annuelle } \\
\text { (millions m3) } \\
\text { Consom./ hab. } \\
150 \mathrm{l} / \text { jour }\end{array}$ & $\begin{array}{c}\text { Taux } \\
\text { croissance annuel } \\
\text { (en milliers) } \\
\text { pour la période } \\
1985 \text { à } 2015 \text { ** }\end{array}$ & $\begin{array}{l}\text { Prévisions } \\
\text { démograph. } \\
\text { en l'an } 2000 \\
\text { en millions } \\
\text { d'habitants }\end{array}$ & $\begin{array}{l}\text { Prévis. consom. } \\
\text { annuelle en } 2000 \\
\text { (millions m3) } \\
\text { Consom./ hab. } \\
200 \mathrm{~V} / \mathrm{jour}\end{array}$ & $\begin{array}{l}\text { Prévis. } \\
\text { démograph. } \\
\text { en } 2015 \\
\text { (en millions } \\
\text { d'habitants) }\end{array}$ & $\begin{array}{l}\text { Prévis. consom. } \\
\text { annuelle en } 2015 \\
\text { (millions de m3) } \\
\text { Consom./hab. } \\
250 \text { Vjour }\end{array}$ \\
\hline Jordanie & $85 / 2.694$ & 147.497 & 37 & 4.647 & 339.231 & 8.016 & 731.460 \\
\hline E. Arabes Unis & $85 / 1.622$ & 88.804 & 17.8 & 2.113 & 154.249 & 2.753 & 251.212 \\
\hline Bahrein & $87 / 0.416$ & 22.776 & 22 & 0.552 & 40.296 & 0.765 & 69.806 \\
\hline Tunisie & $87 / 7.362$ & 403.070 & 25 & 10.148 & 740.804 & 14.697 & 1.341 .101 \\
\hline Algérie & $87 / 22.972$ & 1.257 .717 & 28.8 & 33.227 & 2.425 .571 & 50.870 & 4.641 .888 \\
\hline Djibouti & $87 / 0.456$ & 24.966 & 27.5 & 0.667 & 48.691 & 1.002 & 91.432 \\
\hline Arabie Saoudite & $86 / 12.006$ & 657.329 & 32.7 & 18.916 & 1.380 .868 & 30.714 & 2.802 .652 \\
\hline Soudan & $86 / 22.932$ & 1.255 .527 & 28.2 & 33.847 & 2.470 .831 & 51.366 & 4.687 .148 \\
\hline Syrie & $86 / 10.612$ & 581.007 & 36.1 & 17.744 & 1.295 .312 & 30.205 & 2.756 .206 \\
\hline Somalie & $86 / 4.760$ & 260.610 & 26.6 & 6.874 & 501.802 & 10.192 & 930.020 \\
\hline Cisjordanie / Gaza & $86 / 1.381$ & 75.610 & 37 & 2.296 & 167.608 & 3.961 & 361.441 \\
\hline trak & $86 / 16.230$ & 888.593 & 312 & 25.326 & 848.798 & 40.153 & 3.663 .961 \\
\hline Oman & $86 / 1.381$ & 72.873 & 30.3 & 1.973 & 144.029 & 3.106 & 283.422 \\
\hline Qatar & $86 / 0.305$ & 16.699 & 27.9 & 0.494 & 36.062 & 0.800 & 73.000 \\
\hline Koweit & $85 / 1.697$ & 92.911 & 27.8 & 2.560 & 186.880 & 3.863 & 352.499 \\
\hline Liban & $85 / 3.019$ & 164.385 & 26 & 4.436 & -323.828 & -6.519 & -504.859 \\
\hline Libye & $86 / 3.955$ & 216.536 & 34 & 6.315 & 460.995 & 10.428 & 951.555 \\
\hline Egypte & $86 / 49.609$ & 2.716 .093 & 30.8 & 66.180 & 4.831 .140 & 90.122 & 8.223.633 \\
\hline Maroc & $82 / 20.419$ & 1.117 .940 & 26 & 32.409 & 2.365 .857 & 47.629 & 4.346 .146 \\
\hline Mauritanie & $86 / 1.900$ & 104.025 & 29 & 2.835 & 206.955 & 4.354 & 397.302 \\
\hline Yemen du sud & $85 / 2.294$ & 125.596 & 30.8 & 3.615 & 263.895 & 5.698 & 519.943 \\
\hline Yemen du nord & $86 / 9.274$ & 507.752 & 31.9 & 14.391 & 1050.543 & 23.045 & 2.102 .856 \\
\hline TOTAL & 197.246 & 10.799 .218 & & 291.565 & 2.1284 .245 & 440.258 & 40.173 .542 \\
\hline
\end{tabular}

* Worldmark encyclopedia of the nations - 1988 - Worldmark Press - L.T.D. - John Wiley and Sons - 7e èd. - New.York. Vols. 2 et 4.

* Le taux de croissance annuel figure dans l'encyclopédie World́lmark selon les prévisions des Nations. Unies de 1985 à l'an 2000, à supposer que ce taux se maintienne jusqu'en 2015, Pour le Liban, le taux a étè estimé en fonction des moyennes de croissance avant et après la guerre civile.

3 La consommation de l'eau à des fins domestiques passera de 10.799 millions de $\mathrm{m}^{3}$ à la fin des années 80 à 21.284 millions de $\mathrm{m}^{3}$ en l'an 2000 et à 40.173 millions de $\mathrm{m}^{3}$ en 2015 , si l'on considère que la consommation par habitant va passer de $150 \mathrm{l} / \mathrm{jour}$ dans les années 80 à 200 l/jour en l'an 2000 et à 250 t/jour en l'an 2015 (tableau 1, page cicontre). Certes, ces taux sont plus que modestes si on les compare à la consommation quotidienne par habitant dans les pays développés, où elle atteint 500 l/jour et parfois 700 dans certaines grandes villes américaines. Ils signifient cependant qu'il nous faut garantir plus de 25 millions de $\mathrm{m}^{3}$ supplémentaires par an pour les seuls besoins domestiques à l'aube de l'an 2015.

4 La consommation domestique de l'eau diffère d'un pays arabe à l'autre, néanmoins il est rare que ces taux soient inférieurs à ce qui apparaît dans le tableau 1. Les chiffres fournis donnent un modeste aperçu de la complexité du problème au niveau des pays arabes dans leur ensemble. Si, de plus; on évoque les besoins en eau destinés à satisfaire la demande agricole et industrielle, on obtiendra des chiffres qui, à proportion du volume d'eau actuellement disponible, sont proprement affolants. Le tableau 2 (page suivante) étudie le cas spécifique de l'État des Emirats Arabes Unis. Le déficit hydrique a été estimé à 355 millions de $\mathrm{m}^{3}$ en 1981. Il devrait atteindre 978 millions de $\mathrm{m}^{3}$ en 2015 , en prenant en compte le développement du secteur agricole suivant la croissance démographique.

5 En d'autres termes, la couverture alimentaire fournie par ce secteur restera ce qu'elle était en 1981, et si ce pays désirait couvrir la totalité de ses besoins alimentaires, comme cela devrait être le cas, son déficit serait alors bien supérieur. Même à supposer 
que l'on puisse compenser les pertes dues à l'évaporation, le déficit hydrique serait encore de 871 millions de $\mathrm{m}^{3}$ à l'aube de l'an 2015. Dans ce domaine, les pays du Golfe pourraient combler leur déficit par le dessalement de l'eau de mer, opération au coût prohibitif... Mais jusqu'à quel point cela est-il envisageable?

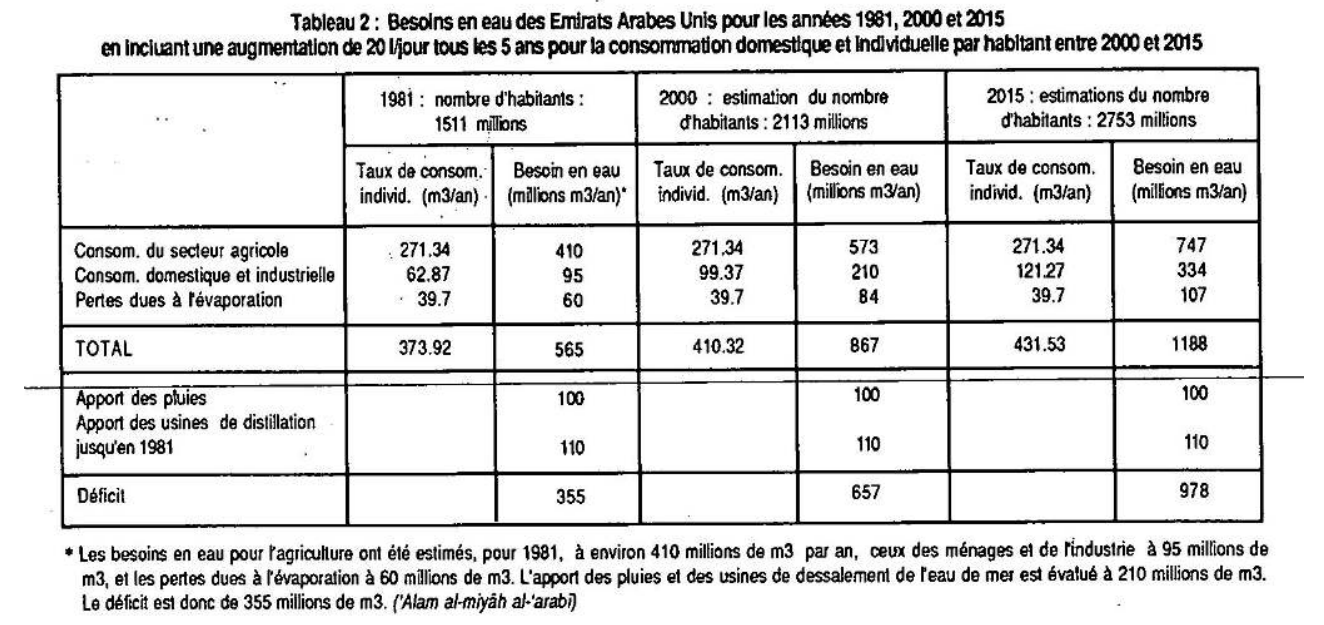

\section{Dessaler l'eau de mer}

Les sommes engagées par les États pour s'approvisionner en eau couvrent le coût d'extension des canalisations, leur entretien, le traitement de l'eau par le chlore, son pompage et son acheminement vers les lieux de consommation. Autres postes de dépenses à inclure; la construction de barrages, de bassins de retenue, le forage de puits artésiens, etc. Récemment est apparu un nouveau procédé d'exploitation des ressources hydriques, le dessalement de l'eau de mer. Diverses techniques peuvent être mises en œuvre, les plus répandues étant la distillation et l'ex-osmose, au coût très élevé : seuls les pays riches sont susceptibles d'y avoir accès, à savoir les pays du Golfe, dépourvus de ressources hydrauliques pérennes suffisantes pour couvrir leurs besoins. Ayant d'abord choisi d'exploiter les eaux souterraines, ils ont décidé par la suite de construire des usines de dessalement de l'eau de mer d'une puissance encore jamais vue auparavant, le point faible de cette option étant son prix de revient considérable et l'éventualité, toujours à envisager, d'une destruction, délibérée ou non, des usines en question.

7 L'Arabie Saoudite est considérée comme le plus grand producteur d'eau distillée du monde. En 1985, la capacité de production de ses usines était de 500 millions de gallons/jour. Les sommes allouées à l'eau par le 3ème plan quinquennal 1980-1985, estimées à 39.602 billions de riyals saoudiens, donnent une idée du coût de l'approvisionnement en eau, en particulier par dessalement de l'eau de mer $^{3}$. Les usines de dessalement fournissent, outre l'eau, une quantité d'énergie électrique jugée tout à fait excédentaire par rapport aux besoins. Elle est estimée à environ 5.000 mégawatts pour la production de 500 millions de gallons/jour.

8 Le tableau 3 (page suivante) fait apparaître que la consommation des ménages atteindra 4.043 millions de $\mathrm{m}^{3}$ par an dans les pays du Golfe si l'on prend comme critère la consommation individuelle dans la ville de Riyad, estimée à 240 litres/jour, auxquels il faut ajouter 20 litres/jour supplémentaires tous les cinq ans. Si ces pays voulaient 
couvrir cette augmentation au moyen du dessalement de l'eau de mer, le coût annuel de l'opération oscillerait entre 2.304 et 2.991 millions de dollars, en ne prenant en compte que le prix de revient de l'énergie requise et sans inclure le coût de construction des usines, lui-même fort élevé. Si, de plus, ces pays décidaient de couvrir partiellement leur consommation industrielle et agricole par dessalement de l'eau de mer, ces chiffres décupleraient.

Tableau 3 : Croissance des dépenses de consommation domestique pour les pays du Golfe entre 1985 et 2015

\begin{tabular}{|c|c|c|c|c|c|}
\hline \multirow{2}{*}{ ETATS } & \multirow{2}{*}{$\begin{array}{l}\text { Consommation } \\
\text { domestique en } 1985 \\
\text { (millions m3) } \\
240 \text { Vjourhab. }\end{array}$} & \multirow{2}{*}{$\begin{array}{l}\text { Estimation de la consom. } \\
\text { domestique en } 2015 \\
\text { (millions de m3) } \\
360 \text { Vjourhab. }\end{array}$} & \multirow{2}{*}{$\begin{array}{l}\text { Ecart de la consom. } \\
\text { domestique entre } \\
1985 \text { et } 2015 \\
\text { (millions de m3) }\end{array}$} & \multicolumn{2}{|c|}{$\begin{array}{l}\text { Coût sugplément. pour la distillation des } \\
\text { quantités nécessaires en } 2015^{\circ} \text { (millions \$) }\end{array}$} \\
\hline & & & & 57 cents par $\mathrm{m} 3^{*}$ & 74 cents par $m 3{ }^{* *}$ \\
\hline $\begin{array}{l}\text { Arabie Saoudite } \\
\text { Koweit } \\
\text { E. Arabes Unis . } \\
\text { Oman } \\
\text { Bahrein }\end{array}$ & $\begin{array}{r}1018.423 \\
144.636 \\
142.087 \\
109.838 \\
-34.065 \\
\end{array}$ & $\begin{array}{r}4.035 .820 \\
507.598 \\
361.744 \\
408.128 \\
-100.521 \\
\end{array}$ & $\begin{array}{r}3.017 .397 \\
362.962 \\
219.657 \\
298.290 \\
\end{array} 65.656$ & $\begin{array}{r}1.719 .916 \\
206.888 \\
125.204 \\
170.025 \\
37.424 \\
\end{array}$ & $\begin{array}{r}2.232 .874 \\
268.592 \\
162.546 \\
220.735 \\
48.585 \\
\end{array}$ \\
\hline $\begin{array}{l}\text { Bahrein- } \\
\text { Qatar }\end{array}$ & $\begin{array}{l}-34.065 \\
25.930\end{array}$ & $\begin{array}{l}-100.521 \\
105.120\end{array}$ & 79.190 & 45.138 & 58.601 \\
\hline TOTAL & 1.475 .779 & 5.518 .931 & 4.043 .152 & 2.304 .595 & 2.991 .933 \\
\hline
\end{tabular}

- En considérant que la tourniture des excédents, jusqưen 2015, ne se tera que par distillation, dans thypothèse ou la majorité des ressources disponibles à ce jour sont utilisées à des fins domestiques, industrielles et agricoles.

* G. Bellort a estimé le coût du m3 d'eau de mer adoucie par distillation à 57 cents si le prix du KWh est à 3 cents - s'il est à 4 cents, le coứt du mélre cube par distillation ou ex-osmose passe à 74 cents, à supposer que la capacié de production de lusine soil d'environ 10 millions de gallonsjour.

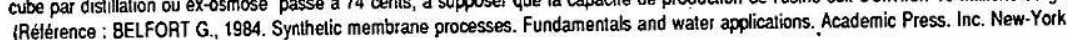

\section{La nappe phréatique}

II existe deux catégories de nappes phréatiques dans les pays arabes: les eaux souterraines renouvelables et les anciennes nappes captives. Les premières sont alimentées par les eaux de pluie. Elles ont, selon les cas, une dimension locale réduite ou, en présence de grands fleuves tels que le Nil, le Tigre ou l'Euphrate, qui irriguent plusieurs pays, une dimension régionale étendue. Certaines nappes phréatiques situées dans les zones arides s'étendent jusqu'à des zones plus arrosées et s'y alimentent pour se prolonger vers d'autres zones arides.

10 Les secondes - les eaux souterraines renouvelables - se trouvent tout naturellement dans les régions soumises à un régime pluvial suffisant. De leurs reliefs montagneux abondamment arrosés jaillissent de riches sources alimentant de nombreux fleuves dont le cours est nourri par les réserves souterraines. Il en est ainsi de l'Algérie, de la Libye, du Liban, de la Jordanie, d'Oman, d'Aden.... Il existe également des eaux souterraines renouvelables dans certaines régions relativement moins arrosées; elles sont peu profondes mais d'excellente qualité.

11 Les eaux souterraines se présentent parfois sous forme de lenticules émergeant audessus du niveau de l'eau salée dans certains pays comme la Libye, sur la plaine côtière égyptienne ou sur celles de la Méditerranée orientale, sur la côte occidentale de l'Arabie, au Bahreïn, au Qatar, à Abou Dhabi, au Koweït, sur la côte orientale des Émirats Arabes Unis. L'intrusion des eaux maritimes dans les nappes souterraines est connue sous le nom de phénomène de Ghyben-Hyrsberg. Ce phénomène est présent partout dans le monde et est lié à un pompage excessif des eaux situées dans les nappes phréatiques côtières. On aboutit alors à une baisse du niveau de ces dernières par rapport au niveau de la mer. L'eau salée se trouve en deçà des côtes, à l'intérieur du désert, sous forme de marais de type marais-salants. 
12 En revanche, de l'eau douce se déverse dans la mer, au-dessous de la surface comme en Méditerranée ou dans le Golfe arabe, et il n'est pas certain que des phénomènes de même type se retrouvent sur les cotes de la mer d'Oman. Ainsi, sur les seules côtes du Liban, on dénombre 26 sources $^{4}$. On en trouve d'autres, dont la source de 'Ayn Zayna près de Benghazi, sur la côte libyenne. Ces sources sont généralement abondantes. On trouve également des eaux souterraines renouvelables dans le lit de fleuves au cours saisonnier, dans des zones faiblement arrosées. L'on sait que les ravins asséchés sont une des conséquences des pluies diluviennes qui se sont abattues lors de périodes géologiques antérieures et sont actuellement comblés de sédiments. On trouve de ces ravins asséchés en Arabie, en Syrie et au nord-est de la Jordanie - anciennement affluents de l'Euphrate, ils lui fournissent encore de temps à autre d'immenses quantités d'eau. Parfois, comme c'est le cas en Arabie, de grandes quantités d'eau douce sont stockées dans la roche endogène ou sédimentaire du ravin. Depuis des temps immémoriaux, ces eaux sont exploitées dans les pays arabes à des fins très diverses, mais leur exploitation excessive, depuis quelque temps, menace de les épuiser, ou bien elles se trouvent polluées au contact des eaux usées. Une étude quantitative aussi bien que qualitative de ces eaux et des masses rocheuses où elles sont retenues en permettrait une meilleure utilisation. Il faudrait comparer les quantités extraites avec leur potentiel de renouvellement et les remplacer artificiellement par des eaux de surface qui se perdent inexorablement vers la mer ou par évaporation.

Quant aux eaux souterraines captives, elles se trouvent dans des réserves relativement profondes et ne sont pas renouvelables par voie naturelle; on pense généralement que l'alimentation de ces sources est tarie depuis 7000 ans au moins ${ }^{5}$. Ces eaux captives sont de trois sortes : douces, salées ou amères. On les trouve :

- En Afrique :

- au Tchad : réserves évaluées à enviro $114.000 \mathrm{Km}^{3}$

- dans le Fezzan : $4.800 \mathrm{Km}^{3}$

- à Koufrah : $3.400 \mathrm{Km}^{3}$

- dans le désert occidental égyptien : $8.000 \mathrm{Km}^{3}$

- en Arabie :

- dans le Noufoud : réserves évaluées environ $4.000 \mathrm{Km}^{3}$

-à Riyad:1.500 Km³

- à Rob al-Khali : $14.500 \mathrm{Km}^{3}$

En incluant ce qui se trouve dans le Sinaï, en Jordanie, en Mauritanie et en Algérie, nous arrivons à un total de plus de $65.000 \mathrm{Km}^{3}$. Ces eaux ont donc une importance considérable du point de vue de la sécurité hydrique des pays arabes et peuvent être considérées comme des réserves et être exploitées dans tous les domaines, les eaux impropres à la consommation pouvant être utilisées dans les puits de pétrole. L'exploitation de ces eaux est d'ailleurs en augmentation puisqu'elles sont utilisées pour l'irrigation du désert et des oasis; mais leur qualité se dégrade au fur et à mesure de leur exploitation en raison de leur inégale densité, les eaux de moindre qualité étant en principe confinées dans les profondeurs de ces bassins souterrains, l'on peut cependant avancer que l'exploitation de ces réserves s'étendra sur plusieurs dizaines d'années. 


\section{Sécurité alimentaire et sécurité hydrique}

15 Stratégiquement parlant, la question de la sécurité alimentaire des pays arabes a supplanté celle de leur sécurité hydrique bien que les deux aspects soient organiquement liés (l'agriculture et l'élevage, source essentielle de l'alimentation, dépendant étroitement de l'approvisionnement en eau).

La consommation en eau diffère selon les cultures envisagées - pérennes ou saisonnières. Elle varie également selon le climat. Ainsi, la consommation hydrique annuelle pour un hectare de culture irriguée est de $7.400 \mathrm{~m}^{3}$ au Liban, quantité qui sera insuffisante dans la plupart des pays arabes eu égard à l'importance du taux d'évaporation, d'une part, et à l'absence de pluie la plus grande partie de l'année, d'autre part. Depuis la construction du barrage sur l'Euphrate, on estime à 640.000 hectares la superficie des terres irriguées dans la région de la Jezireh en Syrie.

On sait que cette dernière consomme annuellement environ 9,2 milliards de $\mathrm{m}^{3}$ d'eau qui proviennent du Tigre et de l'Euphrate. Si l'on considère que cette consommation profite essentiellement au secteur agricole, on peut estimer que cette consommation dépasse $13.000 \mathrm{~m}^{3}$ par an et par hectare. A partir de là, il est facile d'imaginer les quantités d'eau nécessaires pour irriguer l'ensemble des terres cultivables en Arabie Saoudite, par exemple, dont la superficie est évaluée à quelque 4,5 millions d'hectares ${ }^{6}$. Or le volume des eaux de surface, en Arabie seulement, atteint 2040 millions de $\mathrm{m}^{3}$ par an dont $62 \%$ coulent dans la plaine de Tihama ${ }^{7}$.

Aucun pays arabe n'est actuellement autosuffisant en ce qui concerne la couverture de ses besoins alimentaires, alors que certains l'étaient autrefois, comme l'Égypte avant 1970. Cette régression est due la plupart du temps à l'explosion démographique et à l'élévation du niveau de vie. Assurer l'autosuffisance alimentaire pour les 25 prochaines années ne peut donc qu'accentuer le déficit en eau (mises à part les gigantesques réserves d'eau captive) dans les pays arabes qui ne bénéficient pas de ressources hydriques permanentes comme les pays du Golfe, le Yémen, la Jordanie et la Libye. Le problème pourrait concerner par la suite le reste des pays arabes, et il présente d'autres aspects. L'Égypte, par exemple, dépend exclusivement du Nil, qui lui fournit environ 84 milliards de $\mathrm{m}^{3}$ par an. Les terres agricoles ne représentent pour le moment que $3 \%$ de l'ensemble du territoire, sur lesquels vivent $90 \%$ de ta population. Il faut, dès lors, étendre les terres cultivables à d'autres régions et leur fournir l'eau nécessaire, ce qui permettrait, par une nouvelle répartition démographique, de réduire la densité de population de certaines régions et de diminuer la pression économique qui pèse sur elles.

Pour empêcher l'aggravation de la crise de l'eau, il conviendrait de :

- fournir les eaux nécessaires au secteur agricole en s'appuyant sur les techniques les plus modernes. Il faudrait s'inspirer de l'expérience jordanienne, relativement avancée dans ce domaine, et du savoir-faire étranger lorsqu'il est disponible ;

- organiser les diverses cultures selon leur consommation et conformément aux ressources hydrauliques de chaque région ;

- étendre l'autosuffisance alimentaire pour qu'elle profite aux pays arabes dans leur totalité ; pour cela, renoncer à adapter des cultures à des sols qui ne leur conviennent pas, ce qui représente un gaspillage d'immenses potentialités ; 
- profiter au maximum du potentiel hydrique des pays arabes en pompant l'excédent de certains pays pour en faire bénéficier ceux qui en ont besoin, en prenant en compte la distance, les quantités et les conditions techniques.

\section{Le rôle des fleuves}

20 rôle essentiel dans la gestion des besoins en eau. Depuis les années 50, les gouvernements arabes ont édifié des barrages sur ces fleuves et organisé l'exploitation des eaux retenues en amont pour irriguer les terres situées sur leur cours, alimenter les centrales électriques et garantir l'eau potable dans les villes et les villages. Mais jusqu'à ce jour l'exploitation optimale des eaux de surface n'a pas été réalisée dans les pays arabes en dépit de la crise.

21 La construction de barrages et l'élargissement des bassins ne représentent pas toujours la solution idéale: de gigantesques quantités d'eau se perdent par évaporation. Par exemple, un lac d'une superficie de $25 \mathrm{Km}^{2}$ perd 100 millions de $\mathrm{m}^{3}$ par an. Il serait préférable, dans ce cas, d'alimenter des réserves souterraines en creusant des puits jusqu'aux couches susceptibles de stocker l'eau. L'alimentation de telles réserves exige des études précises sur la nature des diverses composantes du terrain, leur perméabilité, leur profondeur et, naturellement, les possibilités de stockage. Il faut ensuite procéder à des comparaisons quantitatives et qualitatives avec les réserves de surface, en prenant en considération les coûts exigés pour chacune des deux opérations.

De gigantesques quantités d'eau - des dizaines de milliards de $\mathrm{m}^{3}$ par an - se perdent également à l'embouchure des fleuves. L'on pourrait envisager de prolonger le lit de ces fleuves pour les faire aboutir là où l'eau manque, ou de pomper l'eau à l'embouchure au moyen de pipe-lines géants en veillant à préserver l'équilibre de l'environnement et à maintenir la navigation sur le fleuve en question. Ces eaux excédentaires à l'embouchure sont d'une nécessité vitale en d'autres lieux et peuvent être exploitées pour l'irrigation, la consommation domestique et la consommation industrielle après traitement. Le coût de cette opération serait nettement inférieur à celui du dessalement de l'eau de mer. Il faut alimenter les nappes phréatiques, dont le niveau ne fait que baisser tandis que leur salinité augmente.

L'embouchure du Chatt al-Arab, où 25 milliards de $\mathrm{m}^{3}$ d'eau sont perdus chaque année, se situe non loin de la partie orientale de l'Arabie Saoudite, des pays du Golfe et de la Jordanie, qui ont un impérieux besoin de ces eaux. La récupération de ces eaux serait incomparablement moins onéreuse que le dessalement et préférables à divers autres projets comme celui des «pipe-lines de la paix» entre la Turquie et les pays arabes. Cette solution éviterait de parier sur la Turquie et ne manquerait pas d'avoir des effets positifs sur Ies régions bénéficiaires. Dans ce domaine, on pourrait utilement profiter de l'expérience libyenne d'extension du fleuve artificiel à travers les régions désertiques, lesquelles présentent une grande similitude avec la région du Golfe. Il serait également possible d'étendre la surface des terres cultivables en Égypte et d'alimenter les réserves souterraines dans la partie nord du désert occidental, non par pompage mais en faisant dériver une partie des eaux excédentaires de l'estuaire jusqu'à la vaste dépression de Qattara, d'une étendue supérieure à $25.000 \mathrm{Km}^{2}$ (au-dessus du niveau de la mer). On pourrait encore, outre l'irrigation et l'alimentation des nappes 
phréatiques, produire d'énormes quantités d'énergie électrique en utilisant la pente des eaux au-dessous du niveau de la mer. Ce projet fournirait à ces zones désertiques de l'eau douce propre à la consommation domestique après traitement. Il est préférable aux projets qui proposent d'amener l'eau de mer vers la dépression pour produire de l'énergie, ce qui risque de provoquer la salinisation progressive de ses eaux - laquelle avoisinerait en peu de temps celle de la mer Morte - et d'exclure par là même toute possibilité d'extension de l'agriculture, non seulement dans cette zone mais également à proximité.

\section{En conclusion}

La question de la sécurité hydrique des pays arabes, abstraction faite de leur sécurité alimentaire, exige donc des réponses rapides et une coopération interarabe inébranlable. Elle ne tolère aucun atermoiement. Il convient :

- d'informer les populations de la gravité du problème, de leur faire comprendre l'importance de l'eau, de les encourager à rationaliser leur consommation dans la mesure du possible et à veiller à la qualité des installations publiques, en particulier les réseaux intérieurs de distribution;

- de traiter les eaux usées pour les réintroduire dans les secteurs adéquats et protéger l'environnement;

- de mener des études sur les eaux de surface, d'une part, et sur les possibilités quantitatives de stockage souterrain pour les eaux renouvelables ou les eaux captives d'autre part ; de proposer les solutions les mieux adaptées pour l'utilisation des eaux de surface; de protéger les eaux souterraines contre te tarissement, la pollution et la salinisation ;

- $d$ 'exploiter les eaux des estuaires pour l'irrigation et la mise en valeur des zones arides et des régions avoisinantes, en réduisant par là même les coûts prohibitifs de techniques telles que le dessalement de l'eau de mer, ce qui éviterait également de dépendre de l'étranger ;

- d'éviter des méthodes archaïques d'irrigation et d'adopter des techniques plus performantes pour la mise en valeur de nouvelles terres agricoles;

- de développer la coopération scientifique et technique entre les pays arabes et de ne recourir au savoir-faire étranger qu'en cas de besoin ;

- d'établir des plans de développement interarabes pour aboutir à l'autosuffisance alimentaire de la nation arabe ;

- de garantir, en qualité et en quantité, la régularité du cours des fleuves prenant leur source à l'étranger, cela en signant traités et accords avec les pays concernés.

\section{NOTES}

1. 1979, «L'essor agricole dans l'État des Emirats Arabes Unis ». 'Alam al-miyâh al-'arabî n¹7, Beyrouth, pp. 8 à 12 .

2. 1981, «L'Arabie Saoudite et l'irrigation du désert ", 'Alam al-miyâh al-'arabî n²7, Beyrouth, pp. 26 à 29. 
3. Centre d'études stratégiques du Golfe, 1989, «L'Arabie Saoudite, le plus grand producteur au monde d'eau distillée ", Al-Hayat du 13 novembre 1989, Londres.

4. ABU CHAKRA (S. F.), 1969, Underground sea water intrusion along the south coast of Lebanon, M. S. Thesis F. Agr. S. C. AUB, Beyrouth.

5. BURDON (D. J.), 1982, Hydrogeological conditions in the Middle East, 9-5-ENG-GEOL. Londres, roi. $15, n^{\circ} 2$.

6. 1978, Reproduction de la publication Middle East Markets. 'Alam al-miyâh al-'arabî nº, Beyrouth, p. 20.

7. NOURY (M.), 1980. «L'eau en Arabie Saoudite », 'Alam al-miyâh al-'arabî n²3, Beyrouth, pp. 17 à 46.

INDEX

Mots-clés : eau, sécurité, stratégie

\section{AUTEURS}

NIZAR AKAR

Université américaine de Beyrouth

\section{TRADUCTEUR_DESCRIPTION}

\section{FRÉDÉRIQUE GOURDAN (TRADUCTION)}

Université américaine de Beyrouth 